
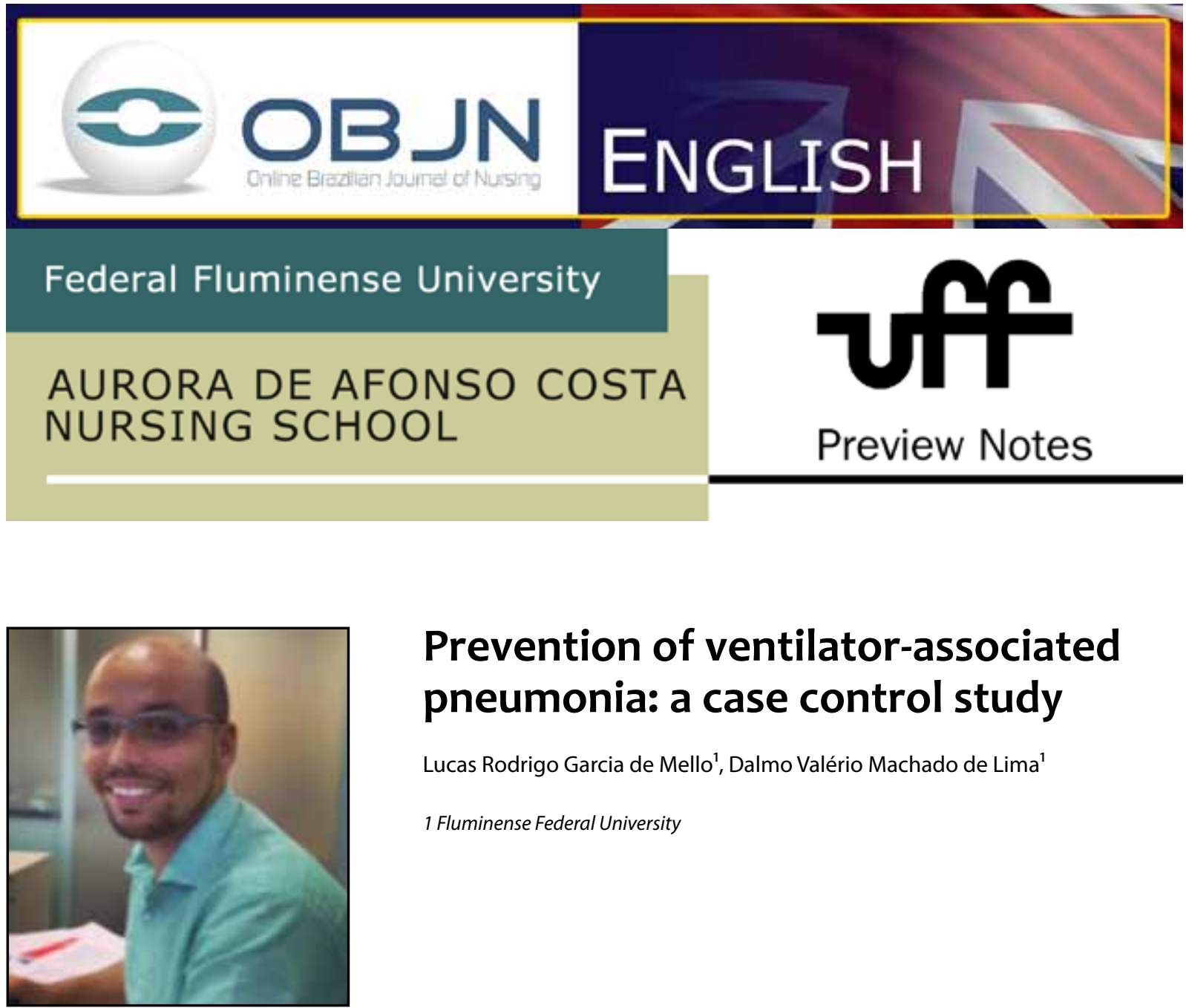

\title{
Prevention of ventilator-associated pneumonia: a case control study
}

\author{
Lucas Rodrigo Garcia de Mello', Dalmo Valério Machado de Lima' \\ 1 Fluminense Federal University
}

\section{ABSTRACT}

Problem: Ventilator-associated pneumonia (VAP) is an infectious process of the pulmonary parenchyma in individuals undergoing mechanical ventilation (MV). Aim: To compare the prevalence of predictor variables among individuals undergoing MV with VAP with the prevalence observed in individuals under MV who do not have the disease. Method: a case-control study with stratified random sample using the medical records of surgical patients undergoing MV between May 2012 and May 2014. The sizing of discrete and finite samples was calculated to get a representative number of the population to be included in the study. The inclusion criteria for the case group is: adult surgical patients under MV who developed VAP. Control group: adult surgical patients undergoing MV for over 48 hours. Exclusion criteria: patients with community and immunosuppressed pneumonia. The SPSS software will be used for processing the data.

Descriptors: Pneumonia, Ventilator-Associated; Ventilators, Mechanical; Nursing Assessment. 


\section{SITUATION AND ITS SIGNIFICANCE}

The Intensive Care Unit (ICU) admits critically ill patients who require continuous specialized professional care, and utilizes specific materials and technology necessary for their diagnosis, monitoring and treatment. Ventilator-associated pneumonia (VAP) is a nosocomial infection with a high impact in terms of mortality rates in intensive care units. In 1,000 hospital discharges, six ventilator-associated pneumonia cases are accounted for in the United States ${ }^{(1)}$. The overall mortality rate in episodes of VAP varies from $20 \%$ to $60 \%$, largely reflecting in these patients the severity of their underlying disease, organ failure and specificities of the studied population and also the etiological agent present ${ }^{(2)}$. Considering the impact of the prevention of VAP in subjects under invasive ventilatory support, this study is relevant to patients because the nursing care for the prevention of VAP will be analyzed. According to the Institute of Health Care Improvement, if applied correctly and to their fullest, these actions - keeping the patient's head elevated at 30 degrees, awaking the patient daily, caring for their oral hygiene and using cuff measurements - can dramatically reduce the incidence of the condition.

This study aims to review the existing protocol in the São José Health Clinic, in Rio de Janeiro, with regard to the prevention of VAP, collaborating with staff in terms of improving clinical nursing practice, and valuing nursing professionals and the institution.

\section{GUIDING QUESTION}

In adult surgical patients under ventilatory assistance who suffer from VAP, how effective is keeping the head lifted, awakening daily, ensuring good oral hygiene and cuff monitoring?
AIM

To compare the prevalence of predictor variables between individuals who developed VAP while undergoing MV with the prevalence observed in individuals who have not developed the disease under MV.

\section{METHOD}

A case-control study with a stratified random sample is used. A retrospective survey of medical records, made between May 2012 and May 2014, of the surgical patients on mechanical ventilation (MV) was performed. From the data, the sizing of a discrete and finite sample was calculated to obtain a representative number for the population included in the study. Inclusion criteria was determined as adult surgical patients undergoing MV who developed VAP. The control group was established as surgical adult patients undergoing ventilatory assistance for more than 48 hours. And as exclusion criteria, community and immunosuppressed pneumonia, as described in the flowchart below:

Figure 1. Flowchart presenting the selection process of subjects for composing the sample of the study. Rio de Janeiro, 2012-2014.

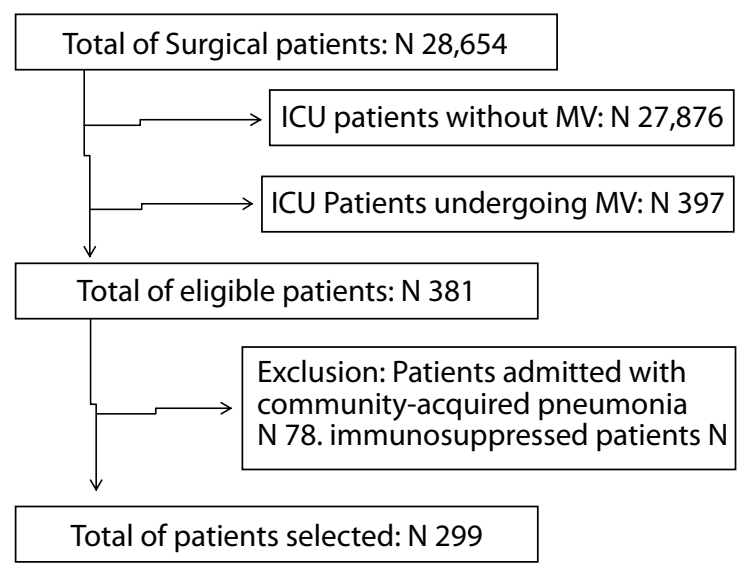

Source: São José Health Clinic, 2014 
The number of adult patients undergoing ventilatory assistance was 299 , and the prevalence of VAP was $14 \%$. Taking these data as the base, the discrete and finite sample was calculated, the representative number for this population being 37 participants. In order to obtain the same proportion of cases and controls according to the calculated $\mathrm{N}$ (37 patients), five cases and 32 controls were selected. The selection will be made from a list of random numbers in terms of the 26 cases, and another list of 160 numbers in terms of controls. The items oral hygiene, $30^{\circ}$ head inclination, daily awakening and cuff measuring will be analyzed. The SPSS software for processing the data will be used, using multiple regression by ordinary least squares equation to relate the occurrence of VAP in a given population to the explanatory variables, i.e., the group of these items set and the individuals undergoing MV. The setting for this study will be the São José Health Clinic in Rio de Janeiro, which has 230 beds, 30 of which are adult ICUs. It was the first hospital in Rio de Janeiro certified as a hospital level I by the National Accreditation Organization (ONA). Currently the hospital is certified as a level III institution by ONA and internationally by the Canadian Council on Health Services Accreditation (CCHSA). This project was approved by the Ethics Committee of the Fluminense Federal University Medical School / FM / UFF / HUAP under opinion ID 643934 issued on 12.05.2014.

\section{REFERENCES}

1. IHI IfHI. How-to guide: Prevent Ventilator - Associated Pneumonia. In: IHI, editor. EUA 2006.

2. Micik S, Besic N, Johnson N, Han M, Hamlyn S, Ball $\mathrm{H}$. Reducing risk for ventilator associated pneumonia through nursing sensitive interventions. Intensive Crit Care Nurs. 2013;29(5):261-5.

3. Lin HL, Lai CC, Yang LY. Critical care nurses' knowledge of measures to prevent ventilator-associated pneumonia. Am J Infect Control. 2014;42(8):923-5.

\begin{abstract}
All authors participated in the phases of this publication in one or more of the following steps, in According to the recommendations of the International Committee of Medical Journal Editors (ICMJE, 2013): (a) substantial involvement in the planning or preparation of the manuscript or in the collection, analysis or interpretation of data; (b) preparation of the manuscript or conducting critical revision of intellectual content; (c) approval of the versión submitted of this manuscript. All authors declare for the appropriate purposes that the responsibilities related to all aspects of the manuscript submitted to OBJN are yours. They ensure that issues related to the accuracy or integrity of any part of the article were properly investigated and resolved. Therefore, they exempt the OBJN of any participation whatsoever in any imbroglios concerning the content under consideration. All authors declare that they have no conflict of interest of financial or personal nature concerning this manuscript which may influence the writing and/or interpretation of the findings. This statement has been digitally signed by all authors as recommended by the ICMJE, whose model is available in http://www. objnursing.uff.br/normas/DUDE_eng_13-06-2013.pdf
\end{abstract}

Received: 08/14/2014
Revised: 08/29/2014
Approved: 08/29/2014 